\title{
"Vínculo Universidad - Empresa: En Relación con el Mundo Globalizado y de Extrema Competencia. Estudio de Caso Universidad Nacional Jorge Basadre Grohmann de Tacna, 2008-2010".
}

\author{
"In Relation to the Globalized World of Extreme Competition. Case \\ Study Jorge Basadre Grohmann National University of Tacna. 2008- \\ 2010".
}

\author{
1 Mgr. Silvia Munarriz O’Phelan
}

\begin{abstract}
RESUMEN:
La presente investigación pretende analizar el Vinculo Universidad Empresa y estudiar su perspectiva en el mundo globalizado y de extrema competencia. En este sentido, el basamento teórico de la investigación se apoyó en la vinculación universidad - empresa y en el proceso de globalización y de extrema competencia. El problema de investigación: ¿Cómo es el Vínculo Universidad-Empresa: en perspectiva con en el Mundo Globalizado y de Extrema Competencia, en la Universidad Nacional Jorge Basadre Grohmann?, tuvo su respuesta en la siguiente hipótesis: El Vinculo Universidad, en relación con el Mundo Globalizado y de Extrema Competencia, en la Universidad Nacional Jorge Basadre Grohmann de Tacna, periodo, es bajo; para la contrastación de la hipótesis se efectuó un estudio de tipo descriptivo, abordado a través de un diseño en el cual se aplicó la recolección de datos relacionados con el tema tratado, utilizando para ello la técnica de la encuesta y como instrumento un cuestionario.
\end{abstract}

Palabras Clave : universidad, empresa, globalización, competencia.

\section{ABSTRACT:}

This research analyzes the University-company Link and studies its perspective in the global world of extreme competition. In this point, the theoretical basis of the research was supported by the university-company and in the process of globalization and of extreme competition. The research question: What is the University-Company Link like: its perspective relative to globalized world of extreme competition in Jorge Basadre Grohmann National University? It was answered in the following hypothesis: The University link in connection to the globalized world of extreme competition in Jorge Basadre Grohmann National University of Tacna, period, is weak. In order for the hypothesis to be contrasted, a descriptive study was carried out, addressed through a design in which the collection of data related to the topic was applied, by using the technique of the survey and a questionnaire as instrument.

Keywords: university, company, globalization, competition.

Mgr. en Docencia Universitaria, Antropóloga. Docente Asociado de la Facultad de Educación, Comunicación y Humanidades de la Universidad Nacional Jorge Basadre Grohmann de Tacna. 


\section{INTRODUCCIÓN}

Ante los profundos cambios sociales, políticos y económicos experimentados en el pasado reciente en el ámbito mundial, regional y nacional, las instituciones de educación superior y empresas peruanas se ven enfrentadas a importantes y diversos desafios de adecuación. Entre las exigencias que se les plantean en esta etapa de su historia; la construcción de vínculos productivos con las empresas, aparece como uno de los principales problemas pendientes de resolución.

Como consecuencia de ello, desde los años ochenta ha estado ocurriendo en las universidades una redefinición de su función social. Ha adquirido creciente importancia la promoción de experiencias de vinculación más estrechas entre la academia y la empresa para la transferencia de conocimientos y la prestación de servicios a usuarios definidos del mercado productivo; actividades que reportan, simultáneamente, recursos financieros para las instituciones universitarias, en un contexto de escasez de fondos públicos.

También el crecimiento de la investigación y el desarrollo sobre todo en la industria moderna de alta tecnología y extremamente competitiva, ha dado origen a una necesidad de vinculación, cada vez más estrecha, entre la universidad y la empresa. En la actualidad, es preocupación permanente de las universidades asumir un papel protagónico y activo, formando mejores profesionales y diseñando programas educativos de muy buen nivel para las empresas en el mercado de trabajo.

Estudiar el vínculo universidad - empresa es un tópico de relevante importancia en los actuales momentos, como producto de la necesidad de contextualizar la labor de estos centros de estudios superiores para proyectar su acción en función de las necesidades prioritarias de sus actores sociales, y lograr al mismo tiempo la tan ansiada relación efectiva con los entes empresariales que de alguna forma tengan conexión con sus intereses.

Por ello en el presente Trabajo titulado "VÍNCULO UNIVERSIDAD - EMPRESA: EN RELACIÓN CON EN EL MUNDO GLOBALIZADO Y DE EXTREMA COMPETENCIA. ESTUDIO DE CASO UNIVERSIDAD NACIONAL JORGE BASADRE GROHMANN DE TACNA, PERÍODO 2008-2010", se pretende determinar el Vínculo Universidad - Empresa en Perspectiva del Mundo Globalizado y de Extrema Competencia; en la Universidad Nacional Jorge Basadre Grohmann.

\section{METODOLOGÍA}

\subsection{Materiales y Métodos}

El presente estudio es de naturaleza descriptiva, ya que se describen situaciones y eventos, cómo es y cómo se manifiesta el evento.

Se adoptó el diseño (no experimental) descriptivo relacional, puesto que no se manipula la variable independiente; y se percibe medir la correlación directa existente entre dos o más conceptos o variables.

\subsection{Población y Muestra}

\subsubsection{Población}

La unidad de análisis de la presente investigación, esta determinada por 5 (cinco) universidades de Tacna.

\subsection{Técnicas e Instrumentos de Recolección de Datos}

\subsubsection{Técnicas}

Las técnica utilizada para la recolección de datos en la presente investigación fue la encuesta.

\subsubsection{Instrumentos para la Recolección de los Datos}

Para llevar a cabo los objetivos propuestos en el presente trabajo, se utilizaron como instrumento, el cuestionario.

\subsection{Técnicas de Análisis de datos}

La información se procesó electrónicamente, utilizando para ello el programa Microsoft Excel versión Vista; y el software SPSS (versión 15) para presentar los Ítems mediante gráficas $\mathrm{y}$, al mismo tiempo estimar las frecuencias absolutas y relativas de cada variable.

\section{RESULTADOS:}

\subsection{ANÁLISIS E INTERPRETACIÓN DE RESULTADOS DEL CUESTIONARIO DE EVALUACIÓN SOBRE EL VÍNCULO UNIVERSIDAD EMPRESA.}

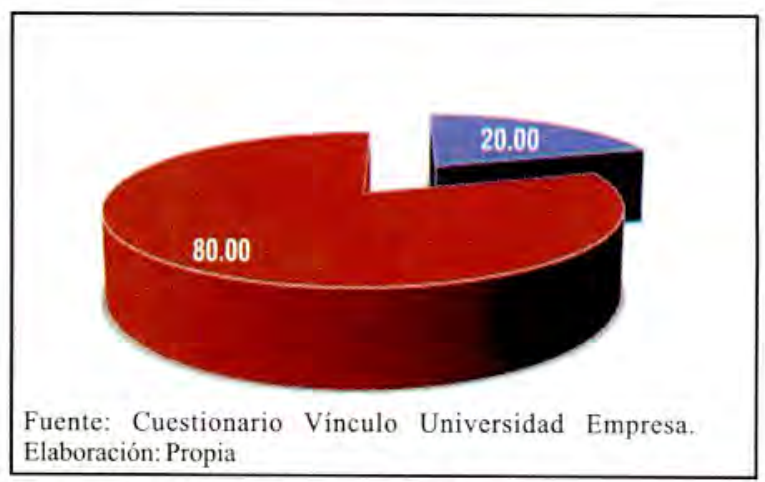

Figura $\mathrm{N}^{\circ}$ 01: Vínculos Universidad-Empresa

El $80 \%$ de los encuestados, no ha incorporado en su programa de desarrollo institucional estrategias para el fomento a la vinculación. Por lo que se observó que surge la gran posibilidad de vínculos Universidad Empresa, para generar recursos que puedan ser compartidos en la misma dinámica del mejoramiento académico y productivo. 


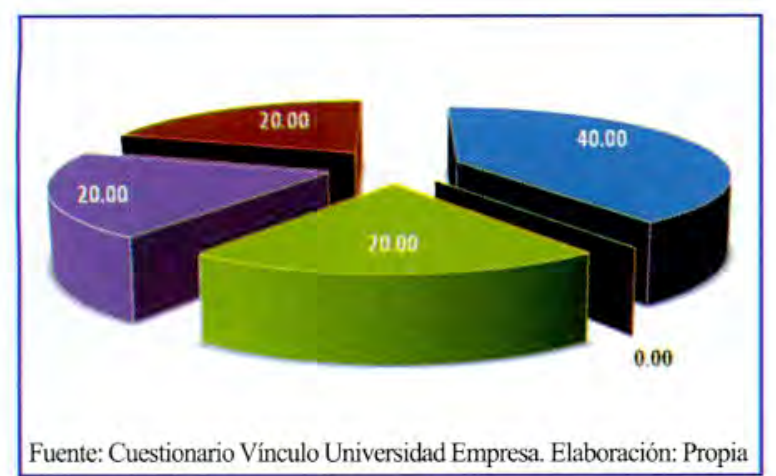

Figura $\mathrm{N}^{\circ} 02$ : Recursos compartidos por la Universidad y la Empresa, en las estrategias de vinculación

Tal como se puede observar en la Tabla y Figura $\mathrm{N}^{\circ} 02$, el $40 \%$ de las universidades hacen uso de recursos compartidos con respecto al factor "humano"; y el $20 \%$ con respecto a los factores intelectuales, técnicos y otros.

Se observó que los recursos compartidos que tienen las universidades en los ámbitos físicos, de la generación y aplicación del conocimiento, desarrollo tecnológico, técnicos y otros, no han logrado generalizarse lo suficiente en apoyo al sector productivo.

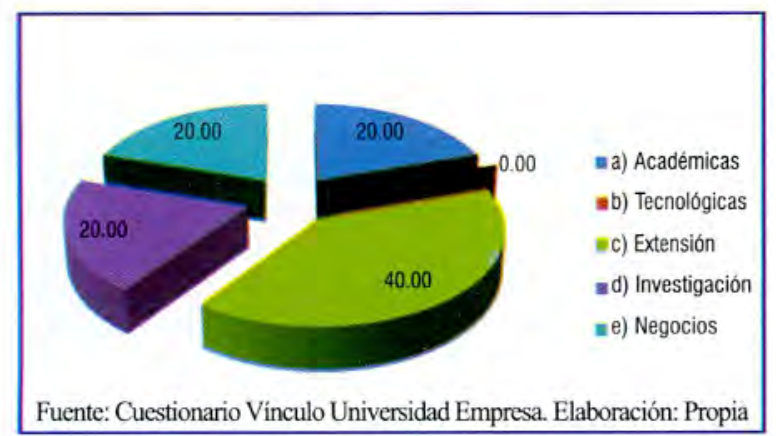

Figura $\mathbf{N}^{\circ}$ 03: Vínculos Universidad - Empresa Actividades Operativas.

El $40 \%$ de las actividades operativas de vinculación que realiza la universidad son de extensión. La extensión universitaria maneja un proceso de comunicación amplio con la empresa y la sociedad, basado en el conocimiento científico, tecnológico, cultural, artístico; y, humanístico.

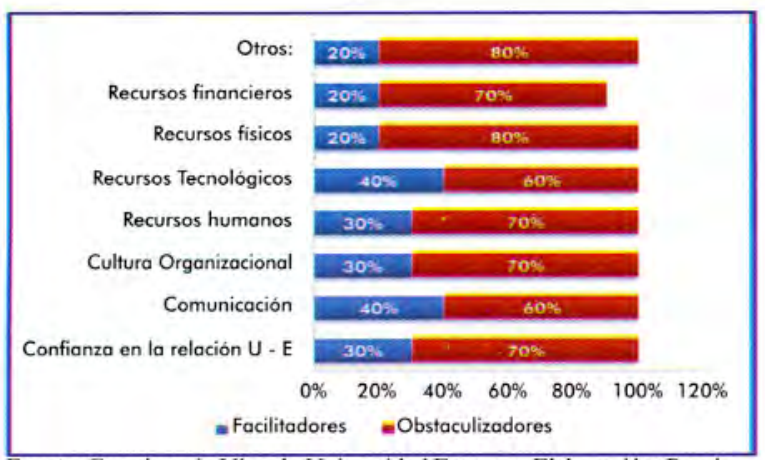

Fuente: Cuestionario Vinculo Universidad Empresa. Elaboración: Propia

Figura $\mathrm{N}^{\circ} \mathbf{0 4}$ : Factores facilitadores y obstaculizadores de los vínculos Universidad - Empresa
El $70 \%$ de las universidades y el $60 \%$ de las empresas indican que existe disponibilidad de los recursos humanos para desarrollar proyectos de vinculación. Esto hace ver que no existe cooperación entre la universidad y la empresa, estas están tratando de generar entre ambos sectores una adecuada complementación y propone algunas pautas para el cambio y el crecimiento tanto de las empresas como de la Universidad. La misión de la empresa y la Universidad es la de incrementar sus relaciones.

Tabla $N^{\circ}$ 01: Vínculos Universidad - Empresa Estrategias de Vinculación. Sector Universidad

\begin{tabular}{|c|c|c|c|}
\hline $\begin{array}{l}\text { ETAPAS DE } \\
\text { ITICTLACTON }\end{array}$ & CARACTERISTICAS & $\begin{array}{c}\text { SECTOR } \\
\text { UNTERSIDAD } \\
\text { (PROMEDIO) }\end{array}$ & $\begin{array}{c}\text { SECTOR } \\
\text { EMPRESA } \\
\text { (PROMEDIO) }\end{array}$ \\
\hline Planeación & $\begin{array}{l}\text { Análisis y detección de las } \\
\text { necesidades de formación. } \\
\text { Planteamiento de estrategias } \\
\text { y politicas. }\end{array}$ & $20 \%$ & $10 \%$ \\
\hline Premociòn & $\begin{array}{l}\text { lnformación, persuasion e } \\
\text { influencia. } \\
\text { Commicación. }\end{array}$ & $30 \%$ & $20 \%$ \\
\hline $\begin{array}{l}\text { Concertación y } \\
\text { Operacién }\end{array}$ & $\begin{array}{l}\text { Formalizar los vinculos. } \\
\text { Ejecución, acciones, } \\
\text { desarrollo, actualización. }\end{array}$ & $10 \%$ & $15 \%$ \\
\hline $\begin{array}{l}\text { Evaluacién } \\
\text { Seguimiento }\end{array}$ & $\begin{array}{l}\text { Verificación de eficiencia, } \\
\text { medición de resultados. } \\
\text { Retroalimentación }\end{array}$ & $10 \%$ & $10 \%$ \\
\hline
\end{tabular}

Fuente: Cuestionario Vínculo Universidad Empresa. Elaboración: Propia

En las diferentes etapas de vinculación se destaca lo siguiente:

\section{Planeación:}

En esta etapa de vinculación no se realizan estudios de detección de necesidades por rama de la actividad productiva, así como prospectivos sobre el comportamiento de las variables económicas asociadas con la producción y el mercado ocupacional. Estos estudios constituyen insumos informativos que permitirán dar respuesta adecuada a los requerimientos del sector empresarial, según sus condiciones y expectativas de productividad e innovación tecnológica, y a las exigencias del desarrollo sustentable que la sociedad plantea a sus sectores productivos.

\section{Promoción}

En cuanto esta etapa se observa lo siguiente: escasa información y comunicación. Por lo que, los que entiendan y practiquen teorías de comunicaciones deben ser capaces de administrar mejor un programa promocional.

\section{Concertación y Operación}

La concertación y operación es parte de la etapa de vinculación en la que existe menor vinculación, por cuanto la universidad se encuentra en una crisis de valores en la que predomina los intereses de grupos frente a los intereses institucionales. Por cuanto se debe hacer énfasis en el Sistema Red de Vinculación alimentado por tres vertientes: los resultados de los 
estudios sectoriales; la identificación de un lenguaje común y la recopilación de información, y se debe sustentar en las ventajas de la tecnología de la información, tanto para su acceso, como para dar respuesta con calidad y oportunidad a los requerimientos de las distintas ramas del sector empresarial, de manera que se logre la interacción equilibrada entre el sector y las capacidades e infraestructura institucional educativa.

Además, en esta etapa, las universidades deben tener presente que se requiere de flexibilidad en lo concerniente al acceso y distribución de los servicios de vinculación tomando en cuenta necesidades específicas de los diversos sectores empresariales. Habrá que diversificar, en estos términos, los tipos y las modalidades de la vinculación, sin que ello signifique demeritar las alternativas, sino por el contrario, sostener un margen básico de calidad de los programas de vinculación que incremente su eficacia y pertinencia al responder a las condiciones sociales, económicas y tecnológicas del sector empresarial.

\section{Evaluación y Seguimiento}

Tomando en cuenta el principio de que no se puede evaluar la calidad si no es mediante la medición de los resultados de cada uno de los elementos, las universidades deben potenciar su que hacer académico con el propósito de alcanzar los niveles de excelencia educativa con base en la normalización.

Hacia el exterior, se debe impulsar esta metodología como mecanismo para lograr la productividad y la calidad que el sector empresarial requiere para enfrentar los retos de la competitividad, en un mundo globalizado.

\section{IV.DISCUSIÓN}

Ante la situación planteada, la vinculación se presenta como una estrategia viable para resolver problemas que aquejan al sector productivo y a las universidades, y tienen la responsabilidad de favorecer y fortalecer el desarrollo de las mismas. Quienes crean que se ha avanzado mucho o suficiente se equivocan. En el mundo actual las empresas compiten en el ámbito planetario. La mentalidad innovadora en aquellos países que apuestan por la educación y la investigación marca una diferencia a favor de sus empresas realmente crucial. A largo plazo, las empresas que mantengan su competitividad deberán hacerlo sobre la base de un capital humano sujeto a formación permanente y en un escenario de innovación continúa únicamente posible con programas de investigación ambiciosos, realistas y ajustados a las necesidades de las empresas.

De igual manera, cabe destacar que para poder afrontar exitosamente la globalización, las empresas requieren, cada vez más, utilizar conocimientos científicos en el proceso productivo $\mathrm{y}$ de gestión, orientados a la reducción de los costos de producción y a aumentar la calidad y el valor de los productos. La reconversión y modernización del aparato productivo no puede lograrse sin una base cientifica y tecnológica. En la medida que las empresas no posean un centro propio de generación, transformación o asimilación sistemática de dichos conocimientos, se ven inducidas a recurrir a las universidades, especialmente las medianas y pequeñas empresas.

Pero la vinculación Universidad-Empresa no solo debe ser beneficiosa para el sector productivo sino permitir también a la Universidad modernizar sus currículos y maximizar los alcances de la investigación que realiza, hasta lograr una sólida capacidad de transferencia científica y tecnológica. El modelo Universidad Empresa permitirá superar el aislamiento académico, la carencia de vinculación en la enseñanza y la investigación poco significativa de la universidad peruana.

Por su parte la tecnología es en un factor determinante en la vida de la empresa; la capacidad de respuesta al cambio, la concentración del esfuerzo y la inteligencia son fundamentales en los mercados globalizados. Esto ha hecho que las empresas establezcan estrategias de innovación, dentro de las cuáles se busca cada vez más la colaboración con instituciones de educación superior.

\section{CONCLUSIONES}

1. En el proceso de vinculación entre los actores universitarios y los actores sociales empresarios existe una contrastación de objetivos, metodologias de trabajos, criterios de validación y difusión de prácticas, actitudes y valores, entre otros, que originan obstáculos o barreras para la cooperación entre ambas partes. Estas dificultades podrían ser de tipo motivacional, producidas por la falta de confianza entre las partes y, al mismo tiempo, por desconocimiento mutuo.

2. El pequeño y mediano empresario, en su mayoría, no tiene conciencia de la necesidad de formación e incluso, tiende a despreciar como poco práctico, todo lo que tenga alguna orientación académica o connotaciones teóricas. Se consideran eminentemente prácticos y son un poco reacios a invertir en tecnología, a buscar ayuda, asesorías.

3. Las políticas de vinculación de la Universidad Nacional Jorge Basadre Grohmann de Tacna con el sector productivo formuladas e implementadas en los últimos años tienen como base un modelo de universidad tradicional que todavía resiste, pese a que los procesos de cambio están presentes. Los tiempos actuales las enfrentan a presiones múltiples que ponen en discusión la vigencia del modelo tradicional de universidad en el que las disciplinas y los campos científicos y profesionales están fundamentalmente orientados hacia adentro.

4. La falta de vinculación de la Universidad Nacional Jorge Basadre Grohmann de Tacna con el sector empresarial no contribuye en la formación integral de las generaciones de jóvenes y trabajadores; en la formación permanente y actualización de los profesionales, obreros y técnicos; en la solución de 
problemas locales, regionales y nacionales; en la formación pedagógica y especializada de la planta docente propia y adjunta; en la innovación y mejoramiento de los procesos que tienen lugar en ambas partes; en la inserción efectiva de la vida cultural, regional y nacional.

\section{REFERENCIAS BIBLIOGRÁFICAS}

1. ANUIES (2004). Asociación Nacional de las Universidades e Instituciones de Educación Superior. México.

2. BATISTAC.R. (2006) Transferencia de Resultados de La Universidad a La Empresa: El Papel de La Universidad en La Innovación de La Empresa Española. Universidad de Las Palmas. Gran Canaria. España.
3. CÓRDOVA, A. (2006).Globalización: Riesgos y oportunidades para Venezuela. En revista NUEVA ECONOMIA, Academia Nacional de Ciencias Económicas.

4. ESCALONA, G. (2005). Vinculación Industria Instituto Universitario

Politécnico. Proyecto de investigación UNEXPO, Venezuela.

\section{Correspondencia:}

Silvia Munárriz O'Phelan

Ciudad Universitaria "Los Granados"

Av. Miraflores s/n. Tacna. Perú

mosilvia@gmail.com 